\title{
LA FIABILIDAD DE LA ASIGNACIÓN GEOGRÁFICA EN LAS ESTADÍSTICAS DE COMERCIO EXTERIOR: AMÉRICA LATINA Y EL CARIBE (1908-1930) *
}

\author{
ANNA CARRERAS-MARÍN \\ Universitat de Barcelona ${ }^{a}$ \\ MARC BADIA-MIRÓ \\ Universitat Oberta de Catalunya ${ }^{\mathrm{b}}$
}

On the Accuracy of the Geographical Assignment of Foreign Trade Statistics. Latin America and the Caribbean (1908-1930)

\begin{abstract}
The statistical accuracy of Historical Foreign Trade Sources has been stated by Federico and Tena (1991) and Tena (1985, 19991 y 1992). This article follows his works in the most suspect field: geographical distribution. We have use Latin American Coal Trade Data among 1908-1930. Most international trade, considering weight, was coal trade; meanwhile it is an ideal product to isolate geographical effects. Statistical disagreements persistence makes us to think this is not a random phenomenon. We have specified an econometric model based on distance. Results show that including geography we can understand statistical disagreements. As a consequence

\footnotetext{
" Received 02/26/2008. Accepted 10/14/2008. Este estudio forma parte del proyecto de investigación Importaciones y modernización económica en América Látina, 1890-1960. BEC200300190MCYT y del proyecto SEJ2007-60445 MCYT ambos dirigido por A. Carreras. Agradecemos a los miembros de este equipo de investigación y a Yolanda Blasco sus comentarios y sugerencias. Agradecemos el apoyo recibido por parte del proyecto BEC2002-01113 dirigido por C. Sudrià, y del proyecto SEJ2005-02498/ECON dirigido por J. Catalán. También agradecemos los comentarios recibidos de J. Baten, M. Flandreau, A. Herranz, A. Tena, D. Tirado y a los participantes en el seminario de historia económica del Departamento de Historia e Instituciones Económicas de la Universidad de Barcelona, donde fue presentado y a los comentarios de tres evaluadores anónimos de esta revista que han ayudado a mejorar el resultado final. Cualquier error es sólo nuestro.

a Departament d'Història i Institucions Econòmiques. Facultat de Ciències Econòmiques i Empresarials. Av. Diagonal 690, 08080 Barcelona, España. annacarrerasmarin@ub.edu.

b Estudis d'Economia i Empresa. Av. Tibidabo, 39-41, 08035, Barcelona, España. mbmiro@tinet.org.
} 
Latin American Sources appear reasonably accurate, considering its geographical pattern.

Keywords: Statistic accuracy, foreing trade Latin America, Caribbean, coal trade, economic geography

JEL Classification: N01, N76, F14

\section{RESUMEN}

La fiabilidad de las estadísticas de comercio exterior a nivel agregado fue analizada detalladamente por Federico y Tena (1991) y Tena (1985, 19991 y 1992). Este artículo sigue el trabajo de estos autores, adentrándose en el ámbito más problemático de la distribución geográfica. Para ello se han usado los datos del comercio de carbón de América Latina y el Caribe entre 1908 y 1930. Por un lado, el carbón resulta un buen representante del comercio internacional, en términos de peso, y por otro lado, precisamente por su peso, se trata de un producto que permite aislar los posibles efectos geográficos en el registro estadístico. La persistencia temporal, tanto del signo como de la magnitud, de las discrepancias estadísticas entre socios comerciales lleva a plantear un modelo econométrico que introduce la distancia como explicación de tales diferencias. Los resultados de la estimación ponen de manifiesto la aceptable calidad de las estadísticas latinoamericanas, en volúmenes, una vez considerados los patrones geográficos de la distribución comercial.

Palabras clave: fiabilidad estadística, comercio exterior, América Latina y el Caribe, comercio de carbón, geografía económica

\section{INTRODUCCIÓN}

La fiabilidad de las fuentes oficiales de comercio exterior, un tema crucial para los estudios del comercio en perspectiva histórica, ha sido objeto de una amplia literatura. Desde los trabajos iniciales de Ricci (1914), a los ya clásicos de Morgernstern (1963) y a las aportaciones más recientes de Federico y Tena (1991) y Tena $(1985,1991,1992)$, la fiabilidad estadística ha sido evaluada a través de su comparabilidad, es decir, a partir de la comparación entre las fuentes oficiales de pares de socios comerciales. Estas aproximaciones se basan en el hecho de que toda exportación es a la vez una importación, por definición, de modo que lo declarado por el exportador y por el importador debería ser, en teoría, coincidente. Sin embargo, el contraste empírico de esta equivalencia teórica rara vez se cumple. Las diferencias entre lo declarado por exportadores e importadores existen en magnitudes nada desdeñables, al menos a primera vista. 
La constatación de tales diferencias llevó a Morgernstern (1963) a desaconsejar absolutamente el uso de las estadísticas oficiales de comercio exterior, como fuente de información económica. A pesar de esta visión pesimista, las estadísticas comerciales siguieron siendo usadas, en buena parte a causa de la ausencia de fuentes alternativas para el estudio del comercio internacional. Las advertencias de Morgernstern sólo sirvieron para que, en el caso de algunos países concretos, se aplicasen sistemas de corrección de los datos, asumiéndose el hecho que algunos países eran más fiables en sus estadísticas históricas que otros. En general, se tendió a asociar desarrollo económico con fiabilidad estadística, de modo que fueron las estadísticas británicas, primero, y las de los EEUU, después, las que fueron escogidas para reconstruir los pasados comerciales de muchos países.

Federico y Tena $(1991)$ y Tena $(1985,1991,1992)$ hicieron un esfuerzo por explicar las discrepancias estadísticas entre socios comerciales a partir de una aproximación agregada, es decir, evaluando el impacto de tales diferencias sobre los datos de comercio total para cada país. Sus resultados, con algunas excepciones para países concretos, arrojaron una luz de optimismo, validando las estadísticas de comercio exterior como fuente de información. Sin embargo, el pesimismo persistía en el nivel desagregado, es decir, en los datos de la distribución del comercio entre socios comerciales. Es en este campo donde se realiza la aportación más novedosa de este trabajo. Un precedente de este planteamiento se encuentra en el trabajo de Ricci (1914), que trataba de comprobar la fiabilidad de los registros internacionales de un solo producto, el trigo, en un solo año, 1909. Este autor consiguió explicar la mayoría de las diferencias encontradas como causa de los costes de transporte y de ciertas diferencias en los criterios de compilación. Sus resultados daban una visión menos pesimista de la distribución de los registros entre socios comerciales y abrían un nuevo camino para comprobar la fiabilidad de los mismos.

El presente estudio continúa con la tradición de evaluar la fiabilidad estadística a través de la comparabilidad entre fuentes de socios comerciales, pero introduce como novedad la redistribución geográfica, partiendo de la idea que lo que aparentemente parecen comportamientos aleatorios son en realidad patrones geográficos. Es decir, que las diferencias entre las fuentes de los exportadores y de los importadores se podrían explicar en parte, a partir de considerar la redistribución espacial de los desajustes. El análisis que se desarrolla pone de manifiesto que la asignación geográfica de los registros de las importaciones es superior a la de las exportaciones, en términos generales y en circunstancias normales, aunque la calidad de las estadísticas de los exportadores sea casi siempre superior.

En primer lugar se tratan las causas de las diferencias entre las estadísticas de los países exportadores y los importadores, además de hacer una descripción sobre la calidad de las fuentes. Un segundo apartado se detiene en las discrepancias procedentes de la asignación geográfica. A continua- 
ción se centra la atención en las estadísticas de comercio exterior de América Latina, y en particular en el comercio del carbón. En el siguiente apartado, se estima un modelo econométrico para detectar las variables que inciden en los desajustes entre fuentes estadísticas. Cierra un último apartado a modo de conclusiones.

\section{LA FIABILIDAD DE LAS ESTADÍSTICAS DE COMERCIO EXTERIOR}

El estudio del comercio internacional depende casi exclusivamente de la información recogida en las estadísticas oficiales de comercio exterior de los distintos países, de modo que su fiabilidad ha generado una literatura de resultados muy contrastados. Como consecuencia del hecho que toda exportación de un país es a la vez una importación para su socio comercial, el comercio existente entre dos países podría estudiarse a partir de los datos recogidos por el exportador, o bien por los datos registrados por el importador. Unos y otros deberían coincidir, siempre y cuando los métodos de confección de las estadísticas de ambos países fueran similares. No fue sino a partir de la Segunda Guerra Mundial que se desarrollaron los sistemas internacionales de homogeneización de las estadísticas oficiales de comercio exterior, lo que ha facilitado a partir de entonces la comparación estadística y, en consecuencia, su verificación. Por otro lado, la ausencia de tales normas internacionales o su incipiente implantación dificulta la comparación para épocas anteriores. Para el período previo a la estandarización internacional, las discrepancias estadísticas entre fuentes oficiales son una realidad a la que no queda más remedio que hacer frente.

El estudio clásico de Allen y Elly (1953) puso de relieve la presencia de cinco factores que determinan que la exportación de un país no sea recogida del mismo modo como importación de su socio comercial:

1) Las distintas definiciones de las mercancías comercializadas.

2) Los distintos niveles de agregación de las mismas.

3) Los variados sistemas de valoración de las mercancías.

4) Los criterios de asignación geográfica del origen de la importación o del destino de la exportación.

5) Los errores, voluntarios o involuntarios, de los agentes comerciales encargados del registro estadístico.

En primer lugar se considera el hecho que cada país utilice distintas definiciones para las mismas mercancías. Por ejemplo, en el caso del sector textil, las alfombras de lana se incluían indistintamente como productos de lana o bien como confecciones textiles. De este modo, al comparar las partidas de productos de lana entre dos países, las cifras no coinciden simplemente por el hecho que un país incluye las alfombras, y el otro las excluye. 
En estrecha relación con ello, otra causa de discrepancias es el nivel de agregación mínimo usado en cada estadística. Si un país ofrece información desagregada hasta llegar al nivel de alfombras de lana pero su socio sólo registra la categoría de productos de lana, sin especificar su composición, la comparación puede no ser coincidente. Este desigual grado de agregación usado por cada país dependía a su vez de la importancia de cada categoría de producto en su comercio exterior.

Distintas definiciones de las mercancías y distintos niveles de agregación de las partidas podrían explicar parte de las discrepancias estadísticas, pero los mayores problemas van asociados a la valoración. Las unidades de peso usadas entre países no siempre eran las mismas, a pesar de lo cual su conversión no resulta tan problemática como la derivada del uso de precios distintos. En primer lugar, las exportaciones suelen valorarse sin incluir los costes de transporte (f.o.b.), mientras que las importaciones suelen incluirlos (c.i.f.). Pero esta regla no ha sido seguida por todos los países ni en todos los períodos ${ }^{1}$. Otro problema añadido procedía de la fijación de precios por parte de las autoridades (precios oficiales) o el uso de los precios declarados por los comerciantes ${ }^{2}$. Los valores oficiales perdían fiabilidad si se mantenían fijos durante largos períodos de tiempo, ya que en ese caso se distanciaban mucho de los precios reales de mercado. La veracidad de los precios declarados dependía a su vez del sistema de recogida de tal información. Una discrepancia adicional en los sistemas de valoración provenía de los tipos de cambio que cada país aplicaba para convertir su comercio exterior a la moneda del país. Como resultado de la interferencia de los costes de transporte, del uso de precios oficiales o declarados y de la aplicación de tipos de cambio distintos, los valores registrados por el país exportador difícilmente coinciden con los del país importador.

Una fuente adicional de discrepancias entre estadísticas era la capacidad de la administración aduanera de recoger la información de forma precisa, ya fuese por omisiones involuntarias o por errores intencionados. En este sentido juegan un papel importante las medidas arancelarias. En el caso de una exportación o importación sujeta a derechos arancelarios se supone que los esfuerzos de los registradores serían mayores que en los flujos comerciales libres de impuestos. Por otro lado, los comerciantes gravados actuarían de forma inversa, tendiendo a minimizar las partidas sujetas a gravamen. En el extremo de este comportamiento se encontraría el contrabando, fenómeno que explica claramente que las estadísticas entre dos países puedan llegar a arrojar cifras absolutamente distintas.

${ }^{1}$ Société des Nations (1928) ofrece un detallado informe de la forma de valoración de las estadísticas de comercio exterior en el mundo.

2 Rubio y Folchi (2005) atribuyen las importantes discrepancias en los valores del comercio exterior entre Argentina y sus socios comerciales, al uso de valoraciones oficiales para fijar los precios de las importaciones. 
A todo ello se añade el problema de la asignación geográfica, cuestión que configura la hipótesis central del presente análisis. Los métodos que seguía cada país para asignar correctamente el lugar de origen de sus importaciones y el destino de sus exportaciones podían ser distintos: la consignación, la producción original para la importación y el consumo final para la exportación, o bien la compra-venta. El hecho de centrar el análisis en las desviaciones geográficas de las estadísticas oficiales de comercio exterior entre distintos países, supone una aportación original al debate sobre la precisión de dichas fuentes de información.

La inclusión de los sesgos geográficos en el análisis de las discrepancias estadísticas entre socios comerciales permite explicar, en parte, los resultados aparentemente arbitrarios obtenidos por el principal estudio en este tema. Morgernstern (1963) cuantificó las discrepancias estadísticas entre países a través de las siguientes fórmulas:

$$
\frac{I_{1}-E_{2}}{I_{1}} \quad \frac{E_{1}-I_{2}}{E_{1}} \quad \frac{B_{1}-B_{2}}{B_{1}}
$$

donde: $\mathrm{I}_{1}=$ Importaciones del Estado A, procedentes del Estado B, en las estadísticas de A, $\mathrm{E}_{1}=$ Exportaciones del Estado A, hacia el Estado B, en las estadísticas de $\mathrm{A}, \mathrm{I}_{2}=$ Importaciones del Estado B, procedentes del Estado $\mathrm{A}$, en las estadísticas de $\mathrm{B}, \mathrm{E}_{2}=$ Exportaciones del Estado $\mathrm{B}$, hacia el Estado $\mathrm{A}$, en las estadísticas de $\mathrm{B}, \mathrm{B}_{1}=$ Balanza comercial de $\mathrm{A}$ con $\mathrm{B}$, en las estadísticas de $\mathrm{A}, \mathrm{B}_{2}=$ Balanza comercial de $\mathrm{A}$ con $\mathrm{B}$, en las estadísticas de $\mathrm{B}$.

Morgernstern aplicó su índice a los años 1909/13, 1928, 1935, 1938, 1948, 1952, 1956 y 1960, para una muestra de países compuesta por: los EEUU, Canadá, Bélgica, Gran Bretaña, Alemania y Francia, países de los cuales cabría esperar mejores resultados en términos de precisión estadística, si fiabilidad y desarrollo económico presentasen una relación positiva. A pesar de ello, los resultados de Morgernstern fueron absolutamente pesimistas. La comparación entre series estadísticas representaba porcentajes extremadamente elevados superando el 25 por cien en numerosos casos. El único resultado positivo, desde su punto de vista, era que estas diferencias se reducían a medida que avanzaba el tiempo, un resultado que se torna descorazonador para el historiador económico.

Pero el problema para Morgernstern no se encontraba sólo en los elevados porcentajes de las discrepancias. Una segunda causa para el pesimismo procedía de la arbitrariedad de los signos. Según el autor, podríamos llegar a aceptar como válidas diferencias de hasta el 25 por cien, atribuyéndolas al factor flete o a las tarifas arancelarias. Si los países fuesen distantes entre sí, podría confortarnos pensar que las importaciones incluyen los costes de transporte, mientras que los datos registrados como exportación los excluyen. Si los países fuesen vecinos, podríamos justificar la diferencia presumiendo que las importaciones incluyen los costes de atravesar una fronte- 
ra. Pero, ¿cómo explicar los signos positivos, es decir, las situaciones en las que lo que declara el exportador supera a lo que el importador dice estar recibiendo?

Para Morgernstern estos inexplicables signos positivos servían para refutar las estadísticas oficiales de comercio exterior, desde cualquier ángulo. Sin embargo, estudios posteriores han puesto de manifiesto que un mayor volumen de comercio registrado como exportación podría ser sintomático de la presencia de contrabando. Esta explicación sería especialmente válida en el caso de fuertes barreras arancelarias. Aquí se propone una respuesta complementaria basada en los sesgos geográficos, aplicable a los casos donde no se puede presumir la presencia del contrabando, debido a la ausencia de aranceles. En este sentido, el presente análisis se sitúa en la línea de los trabajos posteriores al de Morgernstern que han contribuido a recuperar la confianza en la información que nos ofrecen las estadísticas oficiales de comercio exterior.

Federico y Tena $(1991)$ y Tena $(1985,1991,1992)$ han rectificado las valoraciones más pesimistas de Morgernstern (1963), concluyendo que, salvo algunas excepciones para países concretos, las estadísticas oficiales del comercio exterior tienen un grado de fiabilidad aceptable. Para llegar a esta conclusión, los autores utilizan un índice de comparabilidad diseñado explícitamente para superar los problemas de la asignación geográfica de los datos. Dicho índice consiste en dividir el comercio total del país «i» según las estadísticas del país «i», entre la suma de los datos recogidos en las estadísticas de todos sus socios comerciales. La ecuación es la siguiente:

$$
M_{i}=\sum_{i} \frac{M_{i j}}{X_{j i}} \cdot 100 \quad X_{i}=\sum_{i} \frac{X_{i j}}{X_{j i}} \cdot 100
$$

donde M son las importaciones, $i$ es el país de origen de éstas, $j$ el país de destino y X son las exportaciones.

La aplicación de estas ecuaciones a los datos históricos arroja optimismo sobre los datos agregados, mientras que siembra serias dudas sobre los datos desagregados. Los datos de comercio bilateral, entre pares de países, siguen representando la parte menos fiable de las estadísticas de comercio exterior. Si mediante la agregación geográfica se neutralizan los problemas de fiabilidad estadística, ello significa que los problemas realmente importantes se encuentran precisamente en la distribución geográfica. En este ámbito Tena $(1985,1991,1992)$ sugiere la posibilidad de que los importadores sean más precisos en la asignación geográfica que los exportadores. En el presente artículo se explora esta posibilidad y se contrasta su validez en el caso del comercio de carbón con América Latina. 


\section{LA PROBLEMÁTICA DE LA ASIGNACIÓN GEOGRÁFICA, EL CASO DEL CARBÓN}

La hipótesis que se desarrolla en este artículo parte de la suposición que una parte muy importante de las discrepancias entre estadísticas comerciales procede de sesgos sistemáticos en la asignación geográfica y que éstos están en estrecha relación con la distancia, medida a partir de las rutas comerciales. De este modo, la precisión en la asignación geográfica decrecería a medida que aumenta la distancia, a causa de la existencia de un número mayor de países intermediarios. Es decir, que existiría una tendencia a asignar una mayor cantidad de mercancía a los países vecinos, o a los primeros puertos de una ruta marítima, en detrimento de los países más distantes.

Esta hipótesis se fundamenta en la información cualitativa al respecto. Así, por ejemplo, Bourne (1872) puso de relieve este fenómeno para uno de los países con mayor fiabilidad estadística: Gran Bretaña ${ }^{3}$, mientras Platt (1971) lo hizo para el caso latinoamericano ${ }^{4}$. El ejemplo más claro de la hipótesis de la asignación geográfica lo representa Bolivia. De forma similar a lo que ocurre con Suiza o con cualquier otro país sin salida marítima directa, Bolivia apenas aparece en las estadísticas de los países europeos y de los EEUU. ¿Ello significa que Bolivia jamás comerció con Europa o con los EEUU? Más bien lo que ocurrió es que se asignó el comercio boliviano a países intermediarios, en este caso, Chile y Perú. De este modo, el error de asignación en las estadísticas de los países exportadores se pone de relieve cuando se dispone de la información procedente de las estadísticas bolivianas. La información de este país permite explicar las discrepancias con Bolivia, pero también con Chile y Perú. Las estadísticas europeas y norteamericanas infravaloraron el comercio con Bolivia, a la vez que sobrevaloraron su comercio con Chile y Perú. Los errores geográficos con Bolivia son el ejemplo más claro de la hipótesis que se mantiene en el artículo, a través de la cual se sostiene que las estadísticas latinoamericanas tienen mayor fiabilidad de lo que tradicionalmente se les ha reconocido.

Para poder demostrar esta hipótesis ha sido necesario neutralizar previamente el efecto sobre las discrepancias estadísticas procedente de facto-

${ }^{3}$ Bourne en 1872 planteó muy claramente esta problemática: «A ship takes goods from London to Portland in America, to be thence forwarded to Quebec; shall these be deemed as exported to the States or to Canada? Another brings goods from Boulogne, which have been brought by rail from Marseilles, which place they reached from Naples. Are these to be importations from France or Italy?», p. 202.

${ }^{4}$ Los problemas asignativos toman especial relevancia en Platt (1971): «Latin American trade figures in the nineteenth century, in common with those of most other trading nations, were founded on the assumption that the national origin of imports was generally the last port of shipment, while the destination of exports was taken to be the port at which they were landed", p. 119. 
res distintos a la asignación geográfica. Para ello, la estrategia seguida ha consistido en escoger un producto relativamente homogéneo, como lo es el carbón, en la línea de lo que hizo Ricci (1914) con el trigo. De este modo, la problemática derivada de distintas definiciones o agregaciones quedaría minimizada. Para evitar la inclusión de las distorsiones provocadas por los sistemas de valoración, el análisis se ha realizado con los datos en peso (en toneladas). Por otro lado, era necesario encontrar un producto no sujeto a impuestos arancelarios elevados para evitar los problemas del contrabando o la infravaloración de las importaciones, en el caso de declaraciones voluntarias de los comerciantes. Finalmente, para poder estudiar con mayor precisión el fenómeno de la asignación geográfica había que escoger un producto cuyo coste de transporte fuese alto. A la vez el carbón tiene la virtud de ser suficientemente importante y representativo del comercio mundial. Según Isserlis (1938), el comercio de carbón en 1935 era más del 30 por cien del total del volumen transportado por la flota británica. Por todo ello, se ha escogido el comercio del carbón de América Latina y el Caribe con sus principales proveedores: Gran Bretaña y EEUU.

Todas estas consideraciones suponen limitar las conclusiones del trabajo estrictamente al comercio en peso de productos similares al carbón, no pudiéndose extrapolarse los mismos resultados ni al comercio en valores, ni tampoco a productos cuyas discrepancias estadísticas podrían responder en mayor medida a otras cuestiones como los aranceles o los criterios de clasificación estadística. Sin embargo, teniendo en cuenta la gran importancia del carbón en el comercio internacional del período considerado, se explica una parte nada desdeñable de las discrepancias en las estadísticas comerciales de los distintos países analizados, además de ser una buena aproximación a la problemática general ${ }^{5}$.

\section{LAS ESTADÍSTICAS DE AMÉRICA LATINA Y EL COMERCIO DE CARBÓN}

Los países latinoamericanos, enzarzados en frecuentes conflictos regionales después de su independencia, optaron por los ingresos fiscales, procedentes del comercio exterior, como la principal fuente de financiación ${ }^{6}$. La dependencia financiera del comercio exterior explicaría el sumo cuidado con el que se elaboraron las estadísticas oficiales, produciendo un grado razonable de fiabilidad estadística, no relacionado con su nivel de desarrollo económico. La reivindicación de la calidad estadística latinoamericana, dejando de lado las deficiencias en las valoraciones, es una novedad en la

\footnotetext{
5 Para saber más del comercio de carbón entre América Latina y EEUU, Gran Bretaña y Alemania, entre 1890 y 1930, véase Badia-Miró y Carreras-Marín (2008).

${ }^{6}$ Bulmer-Thomas (2003), Coatsworth y Williamson (2004), y Carreras et al. (2003).
} 
literatura, aunque para países concretos algunos autores nos han precedido en esta afirmación ${ }^{7}$.

El comercio de carbón de los países de América Latina comenzó a finales del siglo XIX. La prácticamente total ausencia de este recurso, esencial para la modernización, determinó una gran dependencia de las importaciones exteriores. Tan sólo producían carbón Chile y México desde 1890, Perú desde 1900, Brasil desde 1912 y Venezuela desde 1913. A pesar de ello el aporte de esta producción latinoamericana al consumo regional era muy modesto, entre el 15 y el 29 por cien. Si la dependencia respecto a la importación de carbón era inevitable, no había tampoco demasiada elección respecto a los países proveedores, pudiéndose importar carbón de Gran Bretaña, EEUU (tabla 1) o, en menor medida, de Alemania y Bélgica.

En Rubio y Folchi (2005) se muestra cómo los desajustes entre las exportaciones declaradas por Alemania, Bélgica, Gran Bretaña y los EEUU, y las importaciones correspondientes de los países de América Latina y el Caribe para el carbón, en el año 1925, se reducen significativamente para el conjunto de la región (tabla 2). Las diferencias agregadas son de poco más de 37.000 toneladas, un 0,2 por cien del movimiento total, mientras que para casos puntuales como el de Bolivia, Costa Rica o Guatemala llegan al 80 por cien del total.

El hecho que los desajustes desaparezcan al realizarse la agregación geográfica no es un fenómeno exclusivo del carbón. Distintos estudios, también para el año 1925, demuestran que la fiabilidad de las estadísticas de comer-

TABLA 1

IMPORTACIONES DE CARBÓN PROCEDENTES DE GRAN BRETAÑA Y EEUU, SOBRE IMPORTACIONES TOTALES DE CARBÓN EN AMÉRICA LATINA EN 1925 (TONELADAS)

\begin{tabular}{|l|c|c|}
\hline \multicolumn{1}{|c|}{ Países } & $\begin{array}{c}\text { Importaciones de carbón } \\
\text { de Gran Bretaña y EEUU }\end{array}$ & $\begin{array}{c}\text { Importaciones de carbón } \\
\text { (totales }\end{array}$ \\
\hline Argentina & $2.919 .304(91,8 \%)$ & 3.178 .473 \\
Bolivia & $5.631(35,8 \%)$ & 15.709 \\
Brasil & $1.712 .713(99,1 \%)$ & 1.727 .050 \\
Chile & $247.039(93.6 \%)$ & 264.070 \\
Cuba & $659.389(100 \%)$ & 659.389 \\
México & $65.703(99,8 \%)$ & 65.845 \\
Perú & $35.473(90,4 \%)$ & 39.235 \\
\hline Total América Latina & & \\
(14 países) & $5.663 .400(94,9 \%)$ & 5.968 .104 \\
\hline
\end{tabular}

1 Se incluyen las importaciones de los productores latinoamericanos de carbón.

Fuente: Tabla 2 de Rubio y Folchi (2005), p. 29.

${ }^{7}$ Kunz, S. (2002) para el caso de México. 
TABLA 2

DISCREPANCIAS ESTADÍSTICAS EN LAS IMPORTACIONES DE CARBÓN PROCEDENTES DE GRAN BRETAÑA, EEUU, ALEMANIA Y BÉLGICA, EN AMÉRICA LATINA (TONELADAS, 1925)

\begin{tabular}{|l|c|c|c|}
\hline Países Importadores & $\begin{array}{c}\text { Datos de } \\
\text { losportadores }\end{array}$ & $\begin{array}{c}\text { Datos de } \\
\text { los importadores }\end{array}$ & $\begin{array}{c}\text { Diferencias } \\
(\%)\end{array}$ \\
\hline Argentina & 2.925 .091 & 3.111 .979 & 3 \\
Bolivia & 664 & 6.077 & 80 \\
Brasil & 1.814 .136 & 1.715 .203 & -3 \\
Chile & 195.197 & 253.554 & 13 \\
Cuba & 701.707 & 659.389 & -3 \\
México & 118.643 & 65.746 & -29 \\
Perú & 32.542 & 38.389 & 8 \\
\hline Total América Latina & \multicolumn{3}{|}{} \\
(17 países) & 6.214 .051 & 6.244 .847 & 0,2 \\
\hline
\end{tabular}

Fuente: Tabla 4 de Rubio y Folchi (2005), p. 30.

cio exterior aumenta a medida que se agregan los países por zonas geográficas. Se han encontrado resultados parecidos para el comercio de petróleo, de bienes de equipo, de cemento, de relojes, de automóviles y de textiles (en este caso para 1913) ${ }^{8}$. Todos ellos refuerzan los trabajos de Federico y Tena (1992) y Tena $(1985,1991,1992)$, al situar el problema de la fiabilidad estadística en la asignación geográfica.

Ampliando el período temporal del análisis de 1908 a 1930, en el presente artículo se han desarrollado cálculos de las diferencias absolutas entre las importaciones y las exportaciones, debido a que el interés está en la magnitud de las diferencias entre las estadísticas de comercio exterior, y no en sus ratios, variable mucho más utilizada en este tipo de estudios ${ }^{9}$. Es decir, lo que interesa en el presente trabajo no es que el carbón que Gran Bretaña no declara estar exportando a Bolivia sea un porcentaje insignificante de la estadística británica, mientras que en el caso boliviano pueda representar el 80 por cien, sino las toneladas de carbón exactas que no se asignan a Bolivia y en cambio se están sumando a otros países vecinos intermediarios.

Para Gran Bretaña (gráfico 1), dos de sus socios comerciales, Brasil y Argentina, muestran un signo positivo en el desajuste (mayores exportaciones registradas por Gran Bretaña a Brasil y Argentina, que importaciones

8 Rubio y Folchi (2005), Carreras-Marín (2005), Tafunell (2007), Yánez y Badía-Miró (2007).

9 El uso de las discrepancias en valores absolutos permite medir mejor la dimensión de las discrepancias en su conjunto. Si se considerasen los valores relativos, pequeñas discrepancias en valores absolutos en países pequeños con un comercio reducido indicarían discrepancias muy elevadas que enmascararían el objetivo del presente artículo. 
GRÁFICO 1

DESAJUSTES ENTRE LAS FUENTES DE COMERCIO EXTERIOR DE GRAN BRETAÑA Y AMÉRICA LATINA Y EL CARIBE (TONELADAS DE CARBÓN), 1908-1930. VARIOS PAÍSES

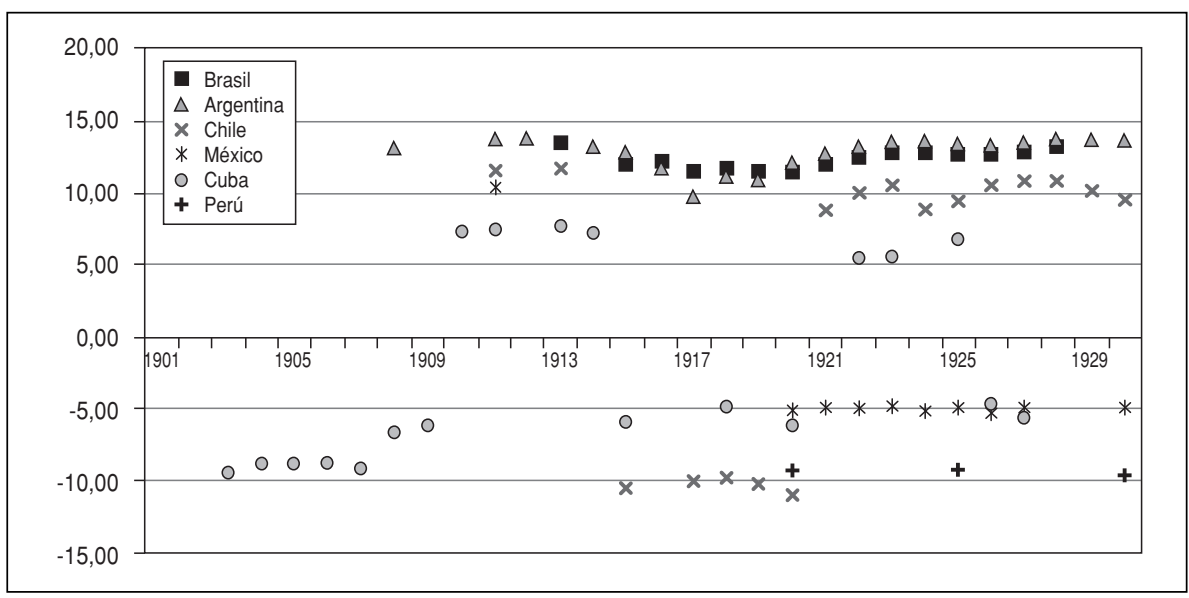

Fuente: Elaboración propia a partir de los Anuarios estadísticos de Comercio exterior. Desajuste medido en $\log (\mathrm{abs}(\mathrm{Xij}-\mathrm{Mji}))$ donde Xij i Mji están expresados en toneladas.

declaradas a la inversa), mientras que otros países como Cuba y México muestran una predominancia de signo negativo (importaciones mayores que las exportaciones registradas por Gran Bretaña). El caso de Chile muestra signo positivo aunque en los años centrales de la Primera Guerra Mundial el signo pasa a ser negativo. Otro dato a tener en cuenta es que a partir de 1925, las exportaciones de carbón a Bolivia procedentes de Gran Bretaña desaparecen, hecho que no se ha podido contrastar con datos de fuentes propias (y por ello no se considera este país en el gráfico 1) ${ }^{10}$.

$\mathrm{El}$ caso de los EEUU es distinto (gráfico 2). Se mantiene el signo positivo para Brasil, México y Argentina, aunque no en todos los casos. Chile ofrece mayores variaciones aunque también se observa una clara permanencia en el signo: positivo durante la Primera Guerra Mundial y negativo después. Por otro lado, las exportaciones de los EEUU hacia Bolivia casi cesan a finales de la década de los veinte, mientras que los datos a partir de fuentes propias siguen creciendo ${ }^{11}$.

Los desajustes obtenidos entre las fuentes de los socios comerciales de América Latina y el Caribe y los dos principales proveedores de carbón,

10 Los datos agregados de importaciones de carbón siguen creciendo en la década de los veinte según la Sociedad de Naciones (1928).

${ }^{11}$ La ausencia de datos continuados para Bolivia ha impedido su inclusión en el gráfico 2 por no ser homogéneos con los datos del resto de países. 
GRÁFICO 2

DESAJUSTES ENTRE LAS FUENTES DE COMERCIO EXTERIOR ENTRE EEUU Y AMÉRICA LATINA Y EL CARIBE (TONELADAS DE CARBÓN), 1908-1930. VARIOS PAÍSES

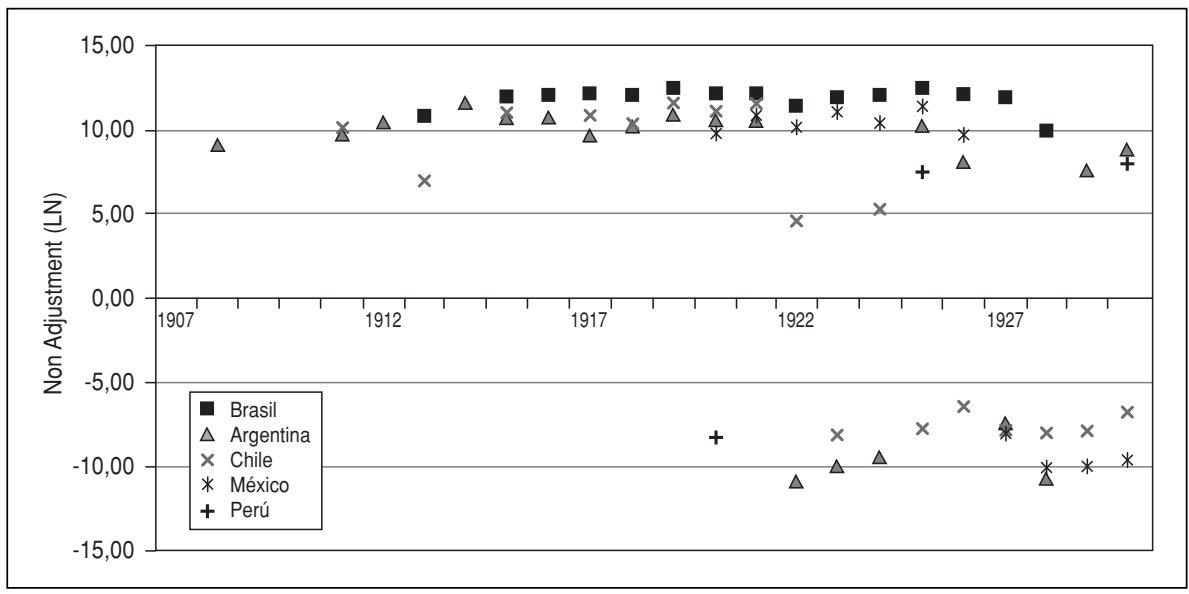

Fuente: Elaboración propia a partir de los Anuarios estadísticos de Comercio exterior. Desajuste medido en $\log (\mathrm{abs}(\mathrm{Xij}-\mathrm{Mji}))$ donde Xij i Mji están expresados en toneladas.

Gran Bretaña y los EEUU, no parecen ser aleatorios (gráficos 1 y 2). Los signos de las discrepancias estadísticas permanecen en el tiempo y la magnitud del error no experimenta drásticas variaciones, aunque tampoco se mantiene inalterada en todo el período. La variable geográfica se pone de relieve con mayor claridad para el caso británico, donde aparecen signos positivos en aquellos países más próximos a Gran Bretaña, a través de las rutas tradicionales de abastecimiento (Brasil era el primer puerto de destino en las rutas británicas hacia América Latina), y signos negativos en los más alejados (Cuba y México) ${ }^{12}$. Esta hipótesis se refuerza por el cambio de signo que tiene México al considerar el comercio de los EEUU, dado que en ese nuevo contexto, este país pasa a ocupar un lugar de mayor proximidad según las rutas comerciales de la época (gráfico 3).

${ }^{12}$ Los criterios de cercanía y de lejanía entre un país y Gran Bretaña se definen a partir del gráfico 3. En él se observa cómo la principal ruta comercial entre América Latina y Gran Bretaña pasaba por Brasil. A partir de allí una parte del comercio se dirigía al Cono Sur y a los países de la costa del Pacífico y la otra se dirigía al Mar del Caribe y a las Antillas. La ruta de los EEUU salía de la Costa Este y seguía por el Mar del Caribe hacia América del Sur, el Canal de Magallanes y la costa del Pacífico (antes de la apertura del Canal de Panamá). 


\section{GRÁFICO 3}

PRINCIPALES RUTAS COMERCIALES EN 1925

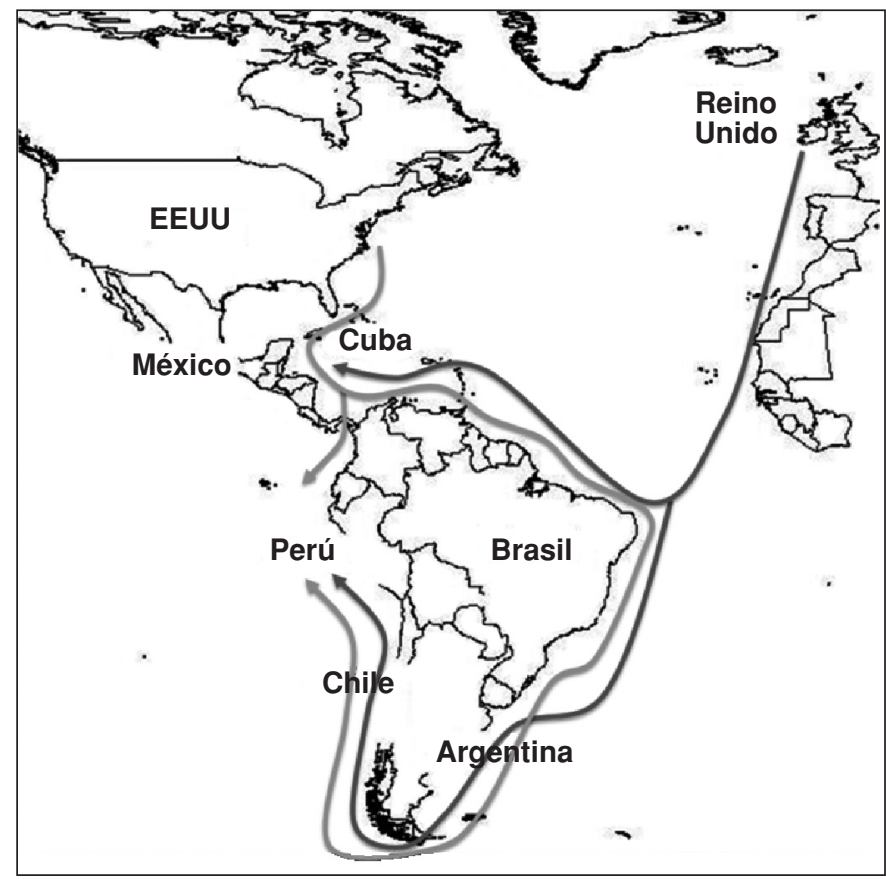

Nota aclaratoria: aunque el canal de Panamá se abrió a la circulación en 1914, el tráfico marítimo que circulaba por esta nueva ruta en 1925 todavía no era de tal magnitud como para tener impacto sobre las grandes rutas comerciales del carbón, motivo por el cual no se ha tenido en cuenta.

Fuente: Berglund, A. (1931). p. 99.

\section{LA ASIGNACIÓN GEOGRÁFICA EN LA ESTIMACIÓN ECONOMÉTRICA DE LAS DISCREPANCIAS ESTADÍSTICAS}

Las dos gráficas anteriores, que muestran la permanencia temporal de los desajustes entre las estadísticas comerciales de América Latina y el Caribe con Gran Bretaña y los EEUU, sugieren que existe un patrón geográfico que explicaría tales diferencias, a través de las rutas comerciales que definen distintas distancias entre socios comerciales. Para comprobar el impacto de la distancia en los desajustes estadísticos se estima un modelo econométrico en el que la ecuación especificada es la siguiente:

$$
\ln \left(\operatorname{NONadj}_{i, j, t}\right)=\alpha_{1}+\alpha_{2} \cdot \ln \left(C O M_{i, t}\right)+\alpha_{3} \cdot \ln \left(D S T_{i, j}\right)+\varepsilon_{i, j, t}
$$


donde: NONadj es la variable dependiente construida a partir de la diferencia entre los datos del exportador y los datos del importador, $C O M$ es la magnitud del comercio, una variable que sirve para neutralizar el efecto del volumen del comercio sobre el desajuste estadístico y $D S T$ es la distancia entre el punto de origen y el de destino, teniendo en cuenta las rutas comerciales habituales ${ }^{13}$.

El signo esperado para $\alpha_{2}$ es positivo, es decir, a mayor volumen de mercancías, más grande será el desajuste. Para $\alpha_{3}$ el signo negativo se corresponde con la hipótesis planteada: es decir, las fuentes de origen estarán sobrestimando los primeros puertos en la ruta frente a la subestimación de los puertos finales. Para el caso que éste sea positivo, tendremos el efecto inverso al planteado: las estadísticas de origen estarán subestimando los primeros puertos en la ruta comercial y sobrestimando los últimos. Es decir, asignarán más carga a los puertos finales en la ruta comercial seguida. En ambos casos, la distancia sirve para explicar las discrepancias, aunque el patrón asignativo difiere radicalmente. Se ha estimado la ecuación (1) por Mínimos Cuadrados Ordinarios. Se han considerado distintas opciones para la distancia, intentando detectar posibles influencias de éstas en la estimación resultante. Los datos de los países de América Latina considerados han sido: Brasil (1915-1928), Argentina (1911-1912 y 1914-1930), Chile (1911, 1913, 1915, 1917-1930), México (1911, 1920-1927), Perú (1920, 1925, 1930) y Cuba (1903-1930).

La primera estimación para Gran Bretaña (GB) considera distancias marítimas directas entre Liverpool y el puerto principal del país de destino. La segunda estimación para Gran Bretaña (GB 2) considera las distancias que siguen las rutas comerciales más importantes de ese momento, es decir, considerando que las distancias a México y Cuba son la suma de las distancias de Gran Bretaña a Brasil y de Brasil a México y Cuba (teniendo en cuenta que la mayor parte del comercio británico tenía en Brasil el primer puerto de destino, en el camino hacia América Latina y el Caribe). En el caso de los EEUU se considera la ruta que bordea América del Sur para llegar a la costa del Pacífico y aquella que va al Pacífico a través del Canal de Panamá. Tal y como se observa en la tabla 3, los resultados en éste último caso no son significativos, por el escaso volumen del tráfico marítimo a través del canal en la mayor parte del período estudiado. La estimación se ha corregido para solucionar los problemas de heteroscedasticidad que generaban las estimaciones por MCO.

En todos los casos, las variables explicativas son significativas y permiten afirmar la verosimilitud del modelo para explicar los desajustes de las estadísticas de comercio exterior. Es decir, parece innegable el impacto que

13 Las distancias no son lineales. Como veremos más adelante, se han considerado distancias marítimas entre los puertos principales de los países de origen y de destino, teniendo en cuenta las rutas comerciales más frecuentes. 
TABLA 3

RESULTADOS DE LA ESTIMACIÓN POR MÍNIMOS CUADRADOS ORDINARIOS DE LA ECUACIÓN 1

\begin{tabular}{|l|c|c|c|c|}
\hline & GB - Dist 1 & GB - Dist 2 & EEUU - Dist 1 & EEUU - Dist 2 \\
\hline CTT & 22,2 & 94,1 & $-17,3$ & $-67,7$ \\
& 0,4 & $1,8^{*}$ & $-0,7$ & $-2,9^{*}$ \\
\hline COM & 2,7 & 1,4 & 1,9 & 1,7 \\
& $6,3^{* *}$ & $2,3^{* *}$ & $4,0^{* *}$ & $6,3^{* *}$ \\
\hline DST & $-5,6$ & $-11,8$ & $-0,2$ & 5,9 \\
& $-1,8^{*}$ & $-2,2^{* *}$ & $-0,1$ & $2,1^{* *}$ \\
\hline $\mathrm{R}^{\wedge} 2$-adj & 0,36 & 0,38 & 0,22 & 0,52 \\
\hline $\mathrm{N}$ & 70 & 70 & 66 & 66 \\
\hline
\end{tabular}

En cursiva están los valores del estadísto t. ** significatividad del 5\% y * significatividad del 1\%. CTT es el coeficiente de la constante del modelo. COM es la variable usada para controlar el efecto de la dimensión del flujo realizado de comercio. DST es la distancia entre los puertos de origen y de destino.

tiene la distancia, lo que valida la explicación de la asignación geográfica en las estadísticas de comercio exterior. También resulta explicativa la variable de dimensión, es decir, a mayor flujo comercial mayor diferencia entre las dos estadísticas. La bondad de la explicación no es demasiado elevada fruto del elevado número de factores que distorsionan las estadísticas del comercio exterior $\left(\mathrm{R}^{\wedge} 2=0,38\right.$ para Gran Bretaña y $\mathrm{R}^{\wedge} 2=0,52$ para los EEUU).

Otro elemento a tener en cuenta es el signo de la variable distancia. Mientras que para Gran Bretaña el signo del coeficiente de la distancia es negativo, para los EEUU, éste es positivo. Esto quiere decir que mientras en el primer caso se sobreestiman los valores para los primeros países (Brasil por ejemplo) y se subestiman los valores de los últimos países (como México y Cuba). Para EEUU sucede todo lo contrario, al mostrar coeficientes con signo positivo, sus exportaciones sobreestiman los valores de los países que están más alejados (como Argentina o Chile ${ }^{14}$ ), mientras que subestiman los valores de los países más cercanos (como Cuba y México). En ambos casos existe un patrón geográfico para las discrepancias estadísticas, pero en cada caso éste se produce de forma distinta.

${ }^{14}$ En este artículo la distancia se limita a las distancias marítimas con lo que no se recoge el posible efecto de las redistribuciones dentro del continente, si se pudiesen añadir las distancias terrestres de las rutas de redistribución interiores se podría complementar el análisis que aquí se presenta, explicando probablemente el caso de Chile y Argentina. 


\section{CONCLUSIONES}

La aportación más relevante del artículo consiste en explicar parte de las discrepancias de las estadísticas de las fuentes oficiales de comercio exterior en su faceta más problemática, es decir, en la asignación geográfica de los flujos comerciales bilaterales. En este sentido se apuesta por la fiabilidad de las estadísticas de comercio exterior situándose en la línea de los trabajos de Federico y Tena (1991) y Tena (1985, 1991 y 1992), a los que se añade el análisis de la distribución comercial. La hipótesis que se plantea consiste en suponer que a través de un adecuado patrón geográfico se pueden explicar, al menos en parte, las diferencias que aparecen en las estadísticas entre socios comerciales.

Para contrastar la hipótesis se ha escogido un área geográfica tradicionalmente considerada muy poco fiable, América Latina y el Caribe, y se ha comparado con dos países de reconocida calidad estadística: EEUU y Gran Bretaña. Los resultados sirven para reconsiderar la calidad estadística latinoamericana, tanto en su asignación geográfica como en sus montantes, aunque no en sus valoraciones. Es decir, los registros de las importaciones resultan más fiables en su asignación geográfica mientras que las estadísticas de los exportadores resultan claramente más fiables en las valoraciones agregadas.

Se ha realizado el análisis de los sesgos estadísticos para el caso concreto del comercio de carbón. El uso de este producto, en términos de peso, tiene como objetivo aislar el fenómeno de la asignación geográfica como principal causa de las discrepancias estadísticas. Por otro lado, la elección del carbón permite recoger una parte muy importante del comercio mundial, básicamente en peso, con lo que no se trata de un producto más, sino de uno de los principales productos del comercio mundial en el período considerado. Ello no significa que en el caso de otros productos comercializados la influencia de la asignación geográfica, como factor explicativo de las diferencias en las estadísticas de los socios comerciales, se vea superada por factores adicionales, como los sistemas de clasificación, las definiciones de las partidas, los sistemas de valoración o las omisiones de la administración aduanera.

La representación gráfica de las diferencias estadísticas en el período 1908-1930 ha supuesto una primera aproximación a la existencia de un comportamiento no aleatorio, al observarse la permanencia temporal de tales sesgos. En los dos países incluidos, Gran Bretaña y EEUU, se observa la permanencia tanto del signo como de la magnitud de las discrepancias. A partir de esta observación se ha planteado la influencia de la distancia como variable explicativa. La estimación de un modelo econométrico de las discrepancias de las fuentes estadísticas a través de la distancia y de la dimensión del flujo del comercio ha permitido detectar la significación de estas variables para explicar los desajustes. Gran Bretaña y los EEUU se ven 
afectados por patrones geográficos distintos pero en ambos casos la distancia, a través de las rutas comerciales, ayuda a entender las discrepancias estadísticas con sus socios de América Latina y el Caribe.

\section{BIBLIOGRAFÍA}

AlLen y ElLy (1953): «International Trade Statistics». New York.

Badia-Miró, M., y CarReras-Marín, A. (2008): «The First World War and Coal Trade Geography in Latin America and the Caribbean, 1890-1930». Jahrbuch für Geschichte Lateinamerikas, vol. 45. En prensa.

BERglund, A. (1931). «Ocean transportation». New York: Longmans, Green \& Co.

BOURNE, S. (1872): «The Official Trade and Navigation Statistics». Journal of the Royal Statistical Society of London, XXXV, pp. 196-217.

Bulmer-Thomas, V. (2003): «The Economic History of Latin America since independence». Cambrige University Press, Cambrige: GB.

Carreras, A.; Hofman, A. A.; TAFunell, X., y Yáñez, C. (2003): «El desarrollo económico de América Latina en épocas de globalización. Una agenda de investigación». Estudios Estadísticos y Prospectivos, 24, CEPAL.

CARreras, A., y TAFunelL, X. (2005): "Capital goods imports and investment in Latin America, 1913 and 1925». Economic History Society, Annual Conference (University of Leicester), Academic session: IIIE: Latin American Development.

CarReras-Marín, A. (2005): «Geographical effects on the accuracy of textile trade data: an international approach for 1913». UPF Economics \& Business Working Papers, 821.

Coatsworth, J. H., y Williamson, J. G. (2004): «Always Protectionist? Latin American Tariffs from Independence to Great Depression». Journal of Latin American Studies, 36, pp. 205-232.

Federico, G., y TENA, A. (1991): «On the accuracy of foreign trade statistics (19091935): Morgernstern revisited». Explorations in Economic History, 28, pp. 259-273.

ISERLIS, M. A. (1938): «Tramp cargoes, and Freights». Journal of the Royal Statistical Society, vol. CI, pp. 53-154.

KunTZ, S. (2002): «Nuevas series del comercio exterior de México, 1870-1929». Revista de Historia Económica, XX: 2, pp. 213-270.

MoRgERNSTERn, O. (1963): «On the accuracy of economic observations». New Yersey: Princeton.

PlatT, D. C. M. (1971): «Problems in the Interpretation of Foreign Trade Statistics before 1914». Journal of Latin American Studies, vol. 3, núm. 2 (nov.), pp. 119-130.

Ricci, U. (1914): «Sulle divergenze fra statistiche del movimento comérciale». Riforma Sociale, vol. 21.

Rubio, M., y Folchi, M. (2005): «On the Accuracy of Latin American Trade Statistics: a Nonparametric Test for 1925». Working Papers del Departament d'Economia $i$ Empresa de la Universitat Pompeu Fabra, núm. 879.

TAFUnelL, X. (2007): «On the origins of ISI: The Latin American Cement Industry, 1900-1930». Journal of Latin American Studies, vol. 39, Iss. 02, pp. 299-323.

TENA, A. (1985): «Una reconstrucción del comercio exterior español, 1914-1935: la rectificación de las estadísticas oficiales». Revista de Historia Económica, año III, núm. 1, pp. 77-119.

— (1991): «Las estadísticas históricas del comercio internacional (1890-1960): fiabilidad y comparabilidad». Tesis doctoral Universidad de Alcalá de Henares, Madrid. 
- (1992): «Las estadísticas históricas del comercio internacional: fiabilidad y comparabilidad». Servicio de Estudios. Estudios de Historia Económica, Banco de España, núm. 24.

YÁÑEZ, C., y BADÍA-Miró, M. (2007): «Las importaciones de relojes y automóviles en América Latina durante 1925». Revista de Historia Industrial, núm. 35, año XVI.

\section{PUBLICACIONES OFICIALES}

Argentina: Dirección General de Estadística (varios años). Anuario del comercio exterior de la República. Argentina, Buenos Aires.

Brazil: Directoria de Estatistica Comercial (varios años). Commercio exterior do Brasil. Rio de Janeiro.

Chile: Oficina Central de Estadística (varios años). Anuario Estadístico de la República de Chile. Comercio Exterior. Valparaíso.

Cuba: Secretaría de Hacienda (varios años). Comercio Exterior. La Habana.

Germany (varios años). Der Auswärtige Handel Deutschlands. Berlin.

Guatemala: Ministerio de Hacienda y Crédito Público (varios años). Memoria de las labores del Ejecutivo en el ramo de Hacienda y Crédito Público. Guatemala.

México: Departamento de Estadística Nacional (varios años). Anuario Estadístico: Comercio exterior y navegación. México D.F.

Perú: Superintendencia General de Aduanas (varios años). Estadística especial del Perú. Callao.

Société des Nations (1928): Mémorandum sur le commerce international et sur les balances des paiements, 1912-1926, Géneve.

U.S. Department of Commerce (varios años). The Foreign Commerce and Navigation of the United States. Washington D.C.

U.S. Department of Commerce (varios años). Commerce Year Book. vol. II (Foreign Countries). Washington D.C.

United Kingdom: Statistical Office of the Customs and Excise Department (varios años). Annual Statement of the Trade of the United Kingdom with Foreign Countries and Britain possessions. London. 
